\title{
Concise Synthesis of Stemofurans A, C, and Derivatives
}

\author{
Hee Jin Lee, Sung Hong Kim, ${ }^{\S}$ Yong Rok Lee, ${ }^{*}$ Xue Wang, ${ }^{\dagger}$ and Won Seok Lyoo \\ School of Chemical Engineering and Technology, Yeungnam University, Gyeongsan 712-749, Korea \\ *E-mail: yrlee@yu.ac.kr \\ ${ }^{\dagger}$ School of Chemical Engineering \& Food Science, Xiangfan University, Hubei 441053, China \\ \$School of Textiles, Yeungnam University, Gyeongsan 712-749, Korea \\ ${ }^{\S}$ Analysis Research Division, Daegu Center, Korea Basic Science Institute, Daegu 702-701, Korea \\ Received August 21, 2010, Accepted August 30, 2010
}

Key Words: Stemofurans A and C, Suzuki coupling, Pyrano-2-arylbenzofurans

Stemofurans A-F (1-6) are isolated from Stemona collinsae, S. tuberose, and S. peirrei, which are mainly distributed in southeast Asia (Fig. 1). ${ }^{1}$ These plants have been used in traditional Asian medical practices for the treatment of inflammatoryrelated diseases. ${ }^{2}$ The dried root tuber of these plants, "baibu", is listed in the Chinese pharmacopoeia and used to relieve cough. ${ }^{3}$ These plants are also used as antiasthmatics in Vietnamese folk medicine. ${ }^{4}$ Furthermore, compounds isolated from these plants have shown to possess antifungal ${ }^{1 \mathrm{a}}$ and antibacterial activities, ${ }^{5}$ as well as inhibition properties of leukotriene formation. ${ }^{1 b}$ These important biological activities and properties have led to the development of new synthetic approaches to such natural products. The first synthesis of stemofuran A(1) was reported by Pasturel et al. starting from 2-hydroxybenzaldehyde through transformations involving hydroxyl protection and deprotection. ${ }^{6}$ Another total synthesis of stemofuran A(1) was accomplished from phenylboronic acid via a [3,3]-sigmatropic rearrangement as the key step in four steps. ${ }^{7}$ Nevertheless, there is still a demand for a more concise and efficient method for synthesizing the biologically interesting stemofurans A - F. In particular, no total synthesis of natural stemofuran $\mathrm{C}$ (3) has been reported thus far.

This lab reported the total synthesis of naturally occurring (+)-machaeriol B (8) using stemofuran A (1) as a key intermediate, which was prepared from commercially available $O$-phenylhydroxylamine (7) in 3 steps according to the known method shown in Scheme 1. As part of an ongoing study for the development of new synthetic routes to stemofurans A-F,
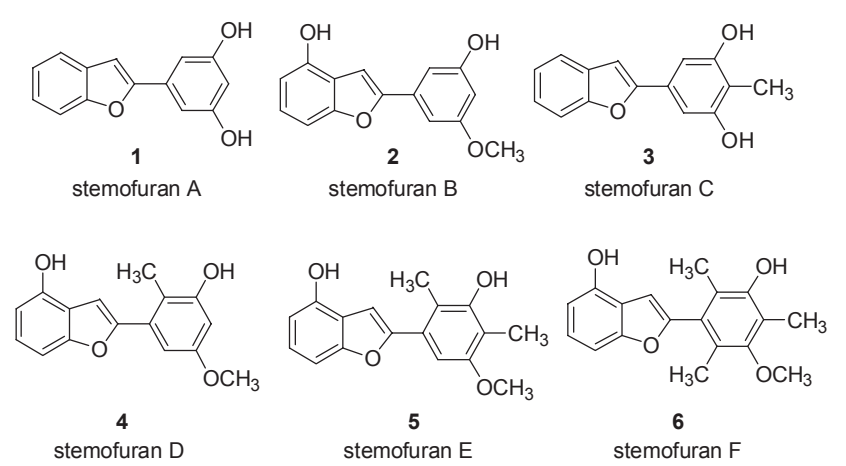

Figure 1. Naturally occurring stemofurans A-F (1-6) isolated from Stemona collinsae. we describe herein a concise synthesis of stemofurans A and C, and its application to give benzofuran molecules with benzopyranyl rings.

Scheme 2 shows the retrosynthetic analysis for stemofurans A (1) and C (3). Stemofuran A (1) can be readily prepared by demethylation of compound 11, generated from commercially available 2-benzofuranboronic acid (9) and 3,5-dimethoxybromobenzene (10) through a Suzuki coupling reaction. In addition, stemofuran C (3) can be prepared from compound 11 by alkylation and demethylation reactions.

Scheme 3 shows a concise synthetic approach to natural stemofurans A (1) and C (3). First, the synthesis of compound $\mathbf{1 1}$ as a key intermediate was attempted using the well-known Suzuki coupling reaction. ${ }^{9}$ Reaction of 2-benzofuranboronic acid (9) with 3,5-dimethoxybromobenzene (10) in the presence of $\mathrm{Pd}\left(\mathrm{PPh}_{3}\right)_{4}$ in refluxing aqueous THF for 8 h gave 11 in $66 \%$ yield. Demethylation of $\mathbf{1 1}$ with $\mathrm{BBr}_{3}$ in methylene chloride at $0{ }^{\circ} \mathrm{C}$ for $5 \mathrm{~h}$ afforded stemofuran $\mathrm{A}(\mathbf{1})$ in $91 \%$ yield. Next, treatment of 11 with $n$-BuLi, followed by addition of methyl iodide, gave 12 in 76\% yield, which was readily converted into stemofuran $\mathrm{C}$ (3) in $92 \%$ yield by treatment with $\mathrm{BBr}_{3}$. The spectroscopic data of synthetic compounds $\mathbf{1}$ and $\mathbf{3}$ are in good agreement with the reported data for the natural products. ${ }^{1 a}$

As an application for usefulness of the synthesized stemofuran

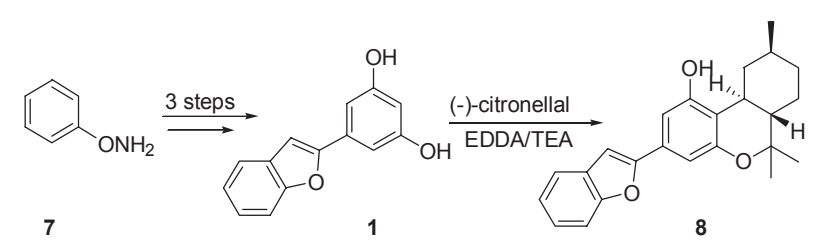

Scheme 1. Reported synthesis of (+)-machaeriol B (8) from stemofuran A (1)

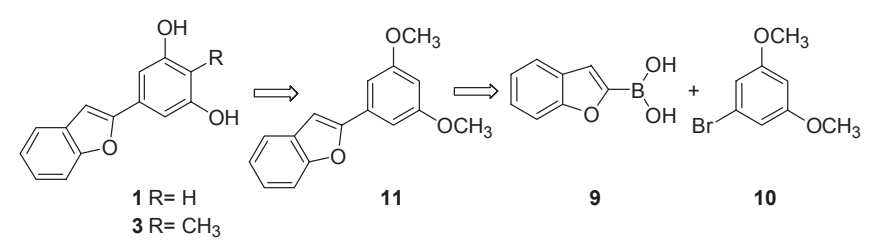

Scheme 2. Retrosynthetic analysis for the synthesis of stemofurans A (1) and C (3) 


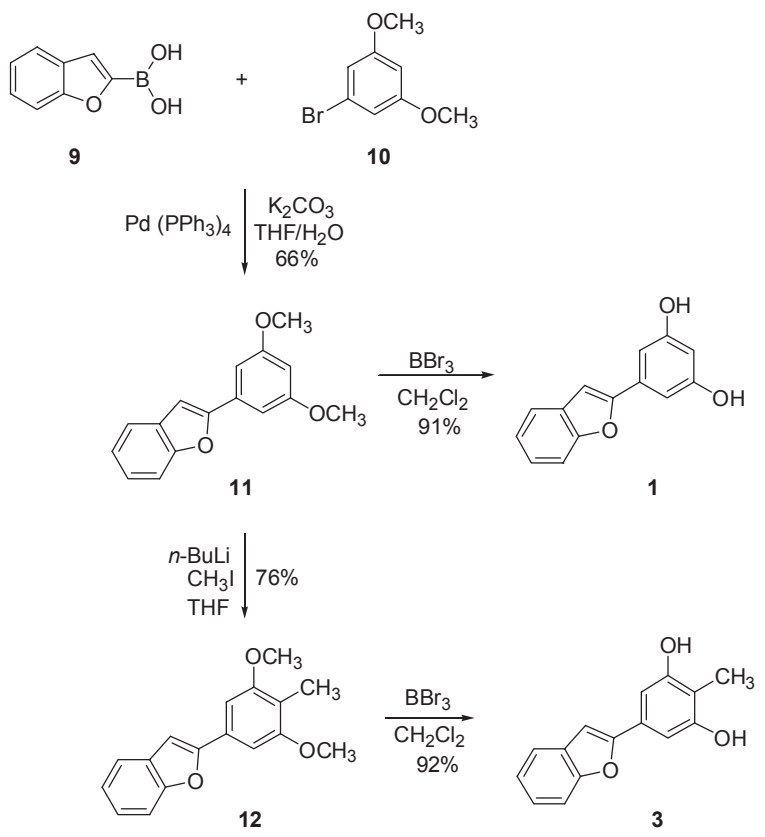

Scheme 3
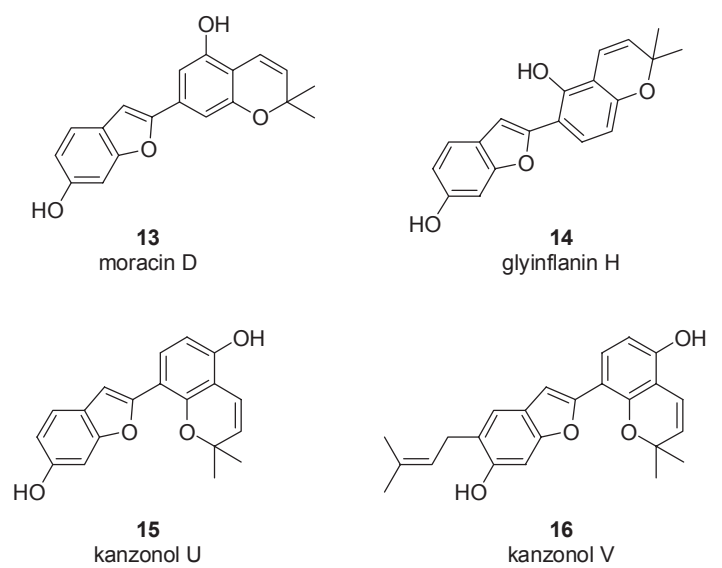

Figure 2. Selected naturally occurring pyrano-2-arylbenzofurans 13-16.

(1), benzopyran formation reactions were next investigated. Benzofuran molecules with benzopyranyl rings (pyrano-2-arylbenzofurans) are widely found in nature ${ }^{10}$ and posses interesting biological activities (Fig. 2). ${ }^{11}$ This range of biological activities and properties has stimulated further research into the synthesis of pyrano-2-arylbenzofuran derivatives.

Recently, we reported a new methodology for synthesizing a variety of benzopyrans by ethylenediamine diacetate-catalyzed reactions of resorcinols with $\alpha, \beta$-unsaturated aldehydes. ${ }^{12}$ Further work and new methodologies for the synthesis of benzofuran molecules with benzopyranyl rings were attempted. Reaction of stemofuran A(1) with 3-methyl-2-butenal was investigated under several catalysts (Table 1). Both indium (III) chloride (20 mol \%) and ytterbium (III) triflate (20 $\mathrm{mol} \%$ ), as Lewis acid catalysts in refluxing acetonitrile, gave no adducts. Treatment of $\mathbf{1}$ with 3-methyl-2-butenal in the presence of 20 $\mathrm{mol} \% \mathrm{Ca}(\mathrm{OH})_{2}$, according to Shigemasa conditions, ${ }^{13}$ gave
Table 1. Reaction of 1 with 3-methyl-2-butenal under several catalysts
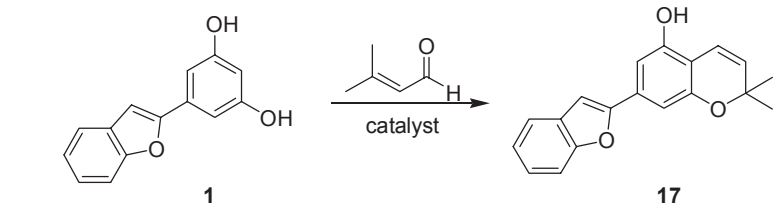

\begin{tabular}{llc}
\hline \multicolumn{2}{c}{ condition } & Yield (\%) \\
\hline $\mathrm{InCl}_{3}(20 \mathrm{~mol} \%)$ & acetonitrile, reflux, 12 h & 0 \\
$\mathrm{Yb}(\mathrm{OTf})_{3}(20 \mathrm{~mol} \%)$ & acetonitrile, reflux, 12 h & 0 \\
$\mathrm{Ca}(\mathrm{OH})_{2}(20 \mathrm{~mol} \%)$ & methanol, reflux, 12 h & 0 \\
pyridine $($ excess $)$ & $140{ }^{\circ} \mathrm{C}, 24 \mathrm{~h}$ & 0 \\
EDDA $(20 \mathrm{~mol} \%)$ & xylene, reflux, 24 h & 30 \\
EDDA $(20 \mathrm{~mol} \%)$ & benzene, reflux, 24 h & 52 \\
\hline
\end{tabular}<smiles>Oc1cc(-c2cc3ccccc3o2)cc2c1C=CC(c1ccccc1)O2</smiles><smiles>CC(C)=CCCC1(C)C=Cc2c(O)cc(-c3cc4ccccc4o3)cc2O1</smiles>

Figure 3

no products. With pyridine as a reactant and solvent, no products were obtained. With ethylenediamine diacetate ( $20 \mathrm{~mol} \%)$ as a catalyst, adduct 17, having a skeleton of the naturally occurring moracin $\mathrm{D}(\mathbf{1 3})$, was produced. The best yield (52\%) was obtained in refluxing benzene for $24 \mathrm{~h}$.

Additional reactions of $\mathbf{1}$ with $\alpha, \beta$-unsaturated aldehydes such as trans-cinnamaldehyde and citral were carried out in the presence of ethylenediamine diacetate (20 mol \%). Reaction of 1 with trans-cinnamaldehyde in refluxing benzene for $24 \mathrm{~h}$ afforded adduct 18 in 51\% yield, whereas with citral, 19 was afforded in $68 \%$ yield (Fig. 3). These reactions provide a rapid route for the synthesis of pyrano-2-arylbenzofuran derivatives.

In conclusion, concise syntheses of biologically interesting stemofurans A (1) and C (3) were carried out starting from 2-benzofuranboronic acid (9) and 3,5-dimethoxybromobenzene (10) in the presence of $\mathrm{Pd}\left(\mathrm{PPh}_{3}\right)_{4}$. The key strategy in the syntheses was the Suzuki coupling reaction. Stemofuran A(1) was readily converted into benzofuran derivatives with benzopyranyl rings. These synthetic routes are expected to be widely used in the synthesis of natural products, including a benzofuran skeleton with benzopyranyl rings.

\section{Experimental Section}

All experiments were carried out in a nitrogen atmosphere. Merck, pre-coated silica gel plates (Art. 5554) with a fluorescent indicator were used for analytical TLC. Flash column chromatography was performed using silica gel 9385 (Merck). ${ }^{1} \mathrm{H}-$ and ${ }^{13} \mathrm{C}$-NMR spectra were recorded on a Bruker Model ARX (300 and $75 \mathrm{MHz}$, respectively) spectrometer in $\mathrm{CDCl}_{3}, \mathrm{CD}_{3} \mathrm{OD}$, and acetone- $d_{6}$ as the solvent chemical shift. All IR spectra were recorded on a Jasco FTIR 5300 spectrophotometer. HRMS and MS spectra were carried out at the Korea Basic Science 
Institute.

2-(3,5-Dimethoxyphenyl)benzofuran (11): To a mixture 2-benzofuranboronic acid ( $0.486 \mathrm{~g}, 3.0 \mathrm{mmol})$, 3,5-dimethoxybromobenzene $(0.651 \mathrm{~g}, 3.0 \mathrm{mmol})$, and $\mathrm{K}_{2} \mathrm{CO}_{3}(0.806 \mathrm{~g}, 4.2$ mmol $)$ in $\mathrm{THF} / \mathrm{H}_{2} \mathrm{O}(1: 1)(30 \mathrm{~mL})$ was added $\mathrm{Pd}\left(\mathrm{PPh}_{3}\right)_{4}(0.175 \mathrm{~g}$, $0.15 \mathrm{mmol}$ ) under $\mathrm{N}_{2}$ and the mixture was heated under reflux for $8 \mathrm{~h}$. The reaction mixture was quenched with saturated $\mathrm{NH}_{4} \mathrm{Cl}$ solution $(30 \mathrm{~mL})$ and extracted with ethyl acetate $(3 \times 30$ $\mathrm{mL})$. The combined extracts were washed with water $(30 \mathrm{~mL})$, dried $\left(\mathrm{MgSO}_{4}\right)$, and evaporated under reduced pressure. Flash chromatography on silica gel using hexane/ethyl acetate $(5: 1)$ afforded $11(0.503 \mathrm{~g}, 66 \%)$ as an oil. ${ }^{1} \mathrm{H}$ NMR $(300 \mathrm{MHz}$, $\left.\mathrm{CDCl}_{3}\right) \delta$ 7.68-7.61 (2H, m), 7.42-7.30 (2H, m), 7.12 (2H, s), 7.09 (1H, s), 6.57 (1H, br s), 3.95 (6H, s); ${ }^{13} \mathrm{C}$ NMR (75 MHz, $\left.\mathrm{CDCl}_{3}\right) \delta 161.1,155.7,154.8,132.2,129.1,124.3,122.9,120.9$, 111.1, 103.0, 101.8, 101.0, 55.4 ; IR (neat) 3063, 2952, 1605, $1459,1354,1250,1202,1158,1067,944,843,747 \mathrm{~cm}^{-1} ;$ HRMS $m / z\left(\mathrm{M}^{+}\right)$calcd for $\mathrm{C}_{16} \mathrm{H}_{14} \mathrm{O}_{3}: 254.0943$. Found: 254.0945.

Stemofuran A (1): To a solution of boron tribromide (2.4 mL, 1.0 M in $\left.\mathrm{CH}_{2} \mathrm{Cl}_{2}, 2.4 \mathrm{mmol}\right)$ in methylene chloride $(30 \mathrm{~mL})$ was added compound $11(0.51 \mathrm{~g}, 2.0 \mathrm{mmol})$ at $0{ }^{\circ} \mathrm{C}$ and the reaction mixture was stirred at $0{ }^{\circ} \mathrm{C}$ for $5 \mathrm{~h}$. Addition of ice water $(30 \mathrm{~mL})$, the mixture was extracted with methylene chloride $(3 \times 30 \mathrm{~mL})$, washed with water, and dried over anhydrous sodium sulfate. Removal of the solvent followed by flash column chromatography on silica gel using hexane/ethyl acetate $(3: 1)$ gave $\mathbf{1}$ $(0.412 \mathrm{~g}, 91 \%)$ as a solid. mp $181-182{ }^{\circ} \mathrm{C} ;{ }^{1} \mathrm{H} \mathrm{NMR}(300 \mathrm{MHz}$, $\left.\mathrm{CD}_{3} \mathrm{OD}\right) \delta 7.46(1 \mathrm{H}, \mathrm{d}, J=8.0 \mathrm{~Hz}), 7.38(1 \mathrm{H}, \mathrm{d}, J=8.0 \mathrm{~Hz})$, 7.18-7.06 (2H, m), $6.93(1 \mathrm{H}, \mathrm{d}, J=1.5 \mathrm{~Hz}), 6.74(2 \mathrm{H}, \mathrm{dd}, J=$ $1.5,1.5 \mathrm{~Hz}), 6.20(1 \mathrm{H}, \mathrm{s}), 4.78(2 \mathrm{H}, \mathrm{br} \mathrm{s}) ;{ }^{13} \mathrm{C} \mathrm{NMR}(75 \mathrm{MHz}$, $\left.\mathrm{CD}_{3} \mathrm{OD}\right) \delta 160.0,157.4,156.1,133.4,130.5,125.3,124.0$, 121.9, 111.8, 104.5, 104.1, 102.3; IR (KBr) 3331, 1620, 1579, 1449, 1358, 1246, 1148, 999, 953, 853, 833, 801, $748 \mathrm{~cm}^{-1}$; EIMS $m / z(\%) 226\left(\mathrm{M}^{+}, 100\right), 197$ (11), 181 (2), 169 (3), 152 (4), 151 (3), 150 (4), 141 (4), 139 (3), 115 (5), 113 (5); HRMS $m / z\left(\mathrm{M}^{+}\right)$calcd for $\mathrm{C}_{14} \mathrm{H}_{10} \mathrm{O}_{3}: 226.0630$. Found: 226.0631 .

2-(3,5-Dimethoxy-4-methylphenyl)benzofuran (12): $n$-BuLi $(0.36 \mathrm{~mL}, 2.5 \mathrm{M}$ in hexane, $0.9 \mathrm{mmol})$ was added at $0{ }^{\circ} \mathrm{C}$ to a solution of $11(0.181 \mathrm{~g}, 0.8 \mathrm{mmol})$ in THF $(20 \mathrm{~mL})$ and the resulting solution was stirred at $0{ }^{\circ} \mathrm{C}$ for $2 \mathrm{~h}$. Methyl iodide $(0.128 \mathrm{~g}$, $0.9 \mathrm{mmol}$ ) was added dropwise to the reaction mixture at $0{ }^{\circ} \mathrm{C}$, which was stirred at room temperature for $10 \mathrm{~h}$. The reaction mixture was quenched with saturated $\mathrm{NH}_{4} \mathrm{Cl}$ solution $(20 \mathrm{~mL})$ and extracted with ethyl acetate $(3 \times 30 \mathrm{~mL})$. The combined extracts were washed with water $(30 \mathrm{~mL})$, dried $\left(\mathrm{MgSO}_{4}\right)$, and evaporated under reduced pressure. Flash chromatography on silica gel using hexane/ethyl acetate (5:1) afforded $12(0.163 \mathrm{~g}, 76 \%)$ as an oil. ${ }^{1} \mathrm{H}$ NMR $\left(300 \mathrm{MHz}, \mathrm{CDCl}_{3}\right) \delta 7.50-7.44(2 \mathrm{H}, \mathrm{m})$, 7.23-7.12 (2H, m), $6.97(2 \mathrm{H}, \mathrm{s}), 6.92(1 \mathrm{H}, \mathrm{br} \mathrm{s}), 3.85(6 \mathrm{H}, \mathrm{s})$, $2.06(3 \mathrm{H}, \mathrm{s}) ;{ }^{13} \mathrm{C} \mathrm{NMR}\left(75 \mathrm{MHz}, \mathrm{CDCl}_{3}\right) \delta 158.5,156.7,154.9$, 129.2, 128.6, 124.1, 122.9, 120.7, 115.0, 111.1, 100.9, 100.4, 55.8, 8.4; HRMS $\mathrm{m} / \mathrm{z}\left(\mathrm{M}^{+}\right)$calcd for $\mathrm{C}_{17} \mathrm{H}_{16} \mathrm{O}_{3}$ : 268.1099. Found: 268.1096.

Stemofuran C (3): To a solution of boron tribromide $(0.6 \mathrm{~mL}$, 1.0 M in $\left.\mathrm{CH}_{2} \mathrm{Cl}_{2}, 0.6 \mathrm{mmol}\right)$ in methylene chloride $(10 \mathrm{~mL})$ was added compound $12(0.134 \mathrm{~g}, 0.5 \mathrm{mmol})$ at $0{ }^{\circ} \mathrm{C}$ and the reaction mixture was stirred at $0{ }^{\circ} \mathrm{C}$ for $10 \mathrm{~h}$. Addition of ice water $(20 \mathrm{~mL})$, the mixture was extracted with methylene chloride
$(3 \times 30 \mathrm{~mL})$, washed with water, and dried over anhydrous sodium sulfate. Removal of the solvent followed by flash column chromatography on silica gel using hexane/ethyl acetate $(3: 1)$ gave $3(0.111 \mathrm{~g}, 92 \%)$ as a solid. mp $195-196{ }^{\circ} \mathrm{C} ;{ }^{1} \mathrm{H}$ NMR $\left(300 \mathrm{MHz}\right.$, acetone- $\left.d_{6}\right) \delta 7.59(1 \mathrm{H}, \mathrm{d}, J=8.1 \mathrm{~Hz}), 7.50(1 \mathrm{H}, \mathrm{d}$, $J=8.1 \mathrm{~Hz}), 7.27-7.22(2 \mathrm{H}, \mathrm{m}), 7.03(1 \mathrm{H}, \mathrm{s}), 6.99(2 \mathrm{H}, \mathrm{s}), 2.14$ $(3 \mathrm{H}, \mathrm{s}) ;{ }^{13} \mathrm{C}$ NMR $\left(75 \mathrm{MHz}\right.$, acetone- $\left.d_{6}\right) \delta 157.5,157.3,155.5$, 100.1, 129.1, 124.9, 123.8, 121.7, 113.0, 111.5, 104.1, 101.4, 8.7; IR (KBr) 3597, 2926, 2855, 1623, 1601, 1577, 1522, 1510, $1453,1421,1377,1365,1351,1299,1257,1185,1157,1144$, $1108,1081,1007,961,937,867 \mathrm{~cm}^{-1}$; HRMS $m / z\left(\mathrm{M}^{+}\right)$calcd for $\mathrm{C}_{15} \mathrm{H}_{12} \mathrm{O}_{3}$ : 240.0786. Found: 240.0788 .

General Procedure for the synthesis of compounds 17-19. Ethylenediamine diacetate $(18 \mathrm{mg}, 0.1 \mathrm{mmol})$ was added to a solution of stemofuran A (1) $(0.5 \mathrm{mmol})$ and $\alpha, \beta$-unsaturated aldehydes $(1.0 \mathrm{mmol})$ in benzene $(10 \mathrm{~mL})$. The reaction mixture was refluxed for $24 \mathrm{~h}$ and the removal of the solvent left an oily residue, which was purified by column chromatography on silica gel to give the products.

Compound 17: A reaction of $1(0.113 \mathrm{~g}, 0.5 \mathrm{mmol})$ with 3-methyl-2-butenal ( $0.084 \mathrm{~g}, 1.0 \mathrm{mmol})$ in refluxing benzene $(10 \mathrm{~mL})$ for $24 \mathrm{~h}$ afforded compound $17(0.076 \mathrm{~g}, 52 \%)$ as an oil. ${ }^{1} \mathrm{HNMR}\left(300 \mathrm{MHz}, \mathrm{CDCl}_{3}\right) \delta 7.72(1 \mathrm{H}, \mathrm{dd}, J=7.5,1.5 \mathrm{~Hz})$, $7.64(1 \mathrm{H}, \mathrm{d}, J=7.5 \mathrm{~Hz}), 7.47-7.36(2 \mathrm{H}, \mathrm{m}), 7.11(1 \mathrm{H}, \mathrm{s}), 7.10$ $(1 \mathrm{H}, \mathrm{s}), 7.04(1 \mathrm{H}, \mathrm{d}, J=1.5 \mathrm{~Hz}), 6.83(1 \mathrm{H}, \mathrm{d}, J=9.9 \mathrm{~Hz}), 5.82$ $(1 \mathrm{H}, \mathrm{d}, J=9.9 \mathrm{~Hz}), 5.32(1 \mathrm{H}, \mathrm{br} \mathrm{s}), 1.64(6 \mathrm{H}, \mathrm{s}) ;{ }^{13} \mathrm{C} \mathrm{NMR}(75$ $\left.\mathrm{MHz}, \mathrm{CDCl}_{3}\right) \delta 155.3,154.7,154.2,151.6,130.9,129.7,129.1$, 124.3, 122.9, 120.9, 116.3, 111.0, 110.1, 105.9, 104.4, 101.6, 76.3, 27.8; IR (neat) 3467, 2974, 2930, 1618, 1562, 1450, 1423, $1370,1251,1120,1069,962,900,849,803 \mathrm{~cm}^{-1}$; HRMS m/z $\left(\mathrm{M}^{+}\right)$calcd for $\mathrm{C}_{19} \mathrm{H}_{16} \mathrm{O}_{3}: 292.1099$. Found: 292.1097.

Compound 18: A reaction of $1(0.113 \mathrm{~g}, 0.5 \mathrm{mmol})$ with transcinnamaldehyde $(0.132 \mathrm{~g}, 1.0 \mathrm{mmol})$ in refluxing benzene $(10 \mathrm{~mL})$ for $24 \mathrm{~h}$ afforded compound $18(0.087 \mathrm{~g}, 51 \%)$ as an oil. ${ }^{1} \mathrm{H}$ NMR (300 MHz, $\left.\mathrm{CDCl}_{3}\right) \delta$ 7.43-7.06 (9H, m), $7.80(1 \mathrm{H}$, $\mathrm{d}, J=10.8 \mathrm{~Hz}), 6.77(1 \mathrm{H}, \mathrm{s}), 6.76(1 \mathrm{H}, \mathrm{s}), 5.79(1 \mathrm{H}, \mathrm{d}, J=3.6$ $\mathrm{Hz}), 5.71(1 \mathrm{H}, \mathrm{dd}, J=10.8,3.6 \mathrm{~Hz}), 5.21(1 \mathrm{H}$, br s $) ;{ }^{13} \mathrm{C} \mathrm{NMR}$ $\left(75 \mathrm{MHz}, \mathrm{CDCl}_{3}\right) \delta 155.1,154.7,154.3,151.6,140.4,131.3$, 129.0, 128.7, 128.4, 127.1, 124.4, 123.6, 122.9, 120.9, 118.1, 111.0, 110.2, 105.6, 104.7, 101.9, 76.9; IR (neat) 2923, 2855, 1626, 1569, 1450, 1357, 1257, 1079, 803, $747 \mathrm{~cm}^{-1}$; HRMS m/z $\left(\mathrm{M}^{+}\right)$calcd for $\mathrm{C}_{23} \mathrm{H}_{16} \mathrm{O}_{3}: 340.1099$. Found: 340.1096 .

Compound 19: A reaction of $1(0.113 \mathrm{~g}, 0.5 \mathrm{mmol})$ with citral $(0.152 \mathrm{~g}, 1.0 \mathrm{mmol})$ in refluxing benzene $(10 \mathrm{~mL})$ for $24 \mathrm{~h}$ afforded compound $19(0.123 \mathrm{~g}, 68 \%)$ as an oil. ${ }^{1} \mathrm{H}$ NMR (300 $\left.\mathrm{MHz}, \mathrm{CDCl}_{3}\right) \delta 7.46(1 \mathrm{H}, \mathrm{d}, J=7.0 \mathrm{~Hz}), 7.38(1 \mathrm{H}, \mathrm{d}, J=7.5$ $\mathrm{Hz}), 7.20-7.12(2 \mathrm{H}, \mathrm{m}), 6.84(2 \mathrm{H}, \mathrm{s}), 6.76(1 \mathrm{H}, \mathrm{s}), 6.61(1 \mathrm{H}, \mathrm{d}$, $J=9.9 \mathrm{~Hz}), 5.52(1 \mathrm{H}, \mathrm{d}, J=9.9 \mathrm{~Hz}), 5.03(1 \mathrm{H}, \mathrm{t}, J=6.6 \mathrm{~Hz})$, 2.12-2.01 (2H, m), 1.78-1.60 (2H, m), $1.58(3 \mathrm{H}, \mathrm{s}), 1.51(3 \mathrm{H}, \mathrm{s})$, $1.34(3 \mathrm{H}, \mathrm{s}) ;{ }^{13} \mathrm{C} \mathrm{NMR}\left(75 \mathrm{MHz}, \mathrm{CDCl}_{3}\right) \delta 155.3,154.6,154.4$, 151.6, 131.7, 130.8, 129.1, 128.7, 124.2, 124.0, 122.8, 120.8, 116.7, 111.0, 110.0, 105.7, 104.3, 101.6, 78.6, 41.0, 26.2, 25.6, 22.7, 17.6; IR (neat) 3410, 3061, 2969, 2823, 1618, 1562, 1449, 1357, 1253, 1157, 1085, 962, 908, 849, 801, $747 \mathrm{~cm}^{-1}$; HRMS $m / z\left(\mathrm{M}^{+}\right)$calcd for $\mathrm{C}_{24} \mathrm{H}_{24} \mathrm{O}_{3}: 360.1725$. Found: 360.1727 .

Acknowledgments. This study was supported by grant No. RTI04-01-04 from the Regional Technology Innovation Pro- 
gram of the Ministry of Knowledge Economy (MKE).

\section{References}

1. (a) Pacher, T.; Segar, C.; Engelmeier, D.; Vajrodaya, S.; Hofer, O.; Greger, H. J. Nat. Prod. 2002, 65, 820. (b) Adams, M.; Pacher, T.; Greger, H.; Bauer, R. J. Nat. Prod. 2005, 68, 83.

2. (a) Bauer, R. In Bioassay Methods in Natural Product and Drug Development; Bohlin, L., Bruhn, J. C., Eds.; Kluwer Academic Publishers: Dorrecht, 1999; p 119. (b) Adams, M.; Kunert, O.; Bauer, R. Planta Med. 2004, 70, 904.

3. Pharmacopoeia of the Peoples Republic of China; Chemical Industry Press: Beijing, 2000; Vol. 1, p 199.

4. Do, T. L. Vietnamese Traditional Medicinal Plants and Drugs, 3rd ed.; Publishing House of Medicine: Hanoi, 2001; p 162.

5. Zhao, W. M.; Qin, G. W.; Ye, Y.; Xu, R. S.; Le, X. F. Phytochemistry 1995, 38, 711 .

6. Pasturel, J. Y.; Solladie, G.; Maignan, J. Fr. Demande FR 2833259 , 2003; Chem. Abstr. 2003, 139, 36375.

7. (a) Miyata, O.; Takeda, N.; Naito, T. Org. Lett. 2004, 6, 1761. (b) Takeda, N.; Miyata, O.; Naito, T. Eur. J. Org. Chem. 2007, 1491.

8. Lee, H. J.; Lee, Y. R.; Kim, S. H. Helv. Chim. Acta 2009, 92, 1404.

9. (a) Miyaura, N.; Suzuki, A. Chem. Rev. 1995, 95, 2457. (b) MetalCatalyzed Cross-Coupling Reactions; Diederich, F., Stang, P. J., Eds.; Wiley-VCH: New York, 1998. (c) Handbook of Organopalla- dium Chemistry for Organic Synthesis; Negishi, E.-I., Ed.; WileyInterscience: New York, 2002.

10. (a) Takasugi, M.; Nagao, S.; Ueno, S.; Masamune, T.; Shirata, A.; Takahashi, K. Chem. Lett. 1978, 1239. (b) Fukai, T.; Nomura, T. Phytochemistry 1995, 38, 759. (c) Fukai, T.; Sheng, C.-B.; Horikoshi, T.; Nomura, T. Pyotochemistry 1996, 43, 1119.

11. Na, M.; Hoang, D. M.; Njamen, D.; Mbafor, J. T.; Fomum, Z. T.; Thuong, P. T.; Ahn, J. S.; Oh, W. K. Bioorg. Med. Chem. Lett. 2007, 17, 3868 .

12. (a) Lee, Y. R.; Choi, J. H.; Yoon, S. H. Tetrahedron Lett. 2005, 46, 7539. (b) Lee, Y. R.; Lee, W. K.; Noh, S. K.; Lyoo, W. S. Synthesis 2006, 853. (c) Lee, Y. R.; Kim D. H. Synthesis 2006, 603. (d) Lee, Y. R.; Kim, J. H. Synlett 2007, 2232. (e) Wang, X.; Lee, Y. R. Tetrahedron Lett. 2007, 48, 6275. (f) Wang, X.; Lee, Y. R. Synthesis 2007, 3044. (g) Lee, Y. R.; Xia, L. Synthesis 2007, 3240. (h) Lee, Y. R.; Kim, Y. M. Helv. Chim. Acta 2007, 90, 2401. (i) Lee, Y. R.; Li, X.; Kim, J. H.; J. Org. Chem. 2008, 73, 4313. (j) Xia, L.; Lee, Y. R. Synlett 2008, 1643. (k) Lee, Y. R.; Hung, T. V. Tetrahedron 2008, 64, 7338. (1) Lee, Y. R.; Xia, L. Tetrahedron Lett. 2008, 49, 3283. (m) Lee, Y. R.; Wang, X. Tetrahedron 2009 , 65, 10125. (n) Lee, Y. R.; Kim, Y. M.; Kim. S. H. Tetrahedron 2009, 65,101 . (o) Jung, D, H.; Lee, Y. R.; Kim, S. H. Helv. Chim. Acta 2010, 93, 635 .

13. Saimoto, H.; Yoshida, K.; Murakami, T.; Morimoto, M.; Sashiwa, H.; Shigemasa, Y. J. Org. Chem. 1996, 61, 6768. 